\title{
MIHÁLY HARSÁNYI
}

\section{DEONYMISCHE WORTBILDUNGSKONSTRUKTIONEN AUF -FRITZE IM DEUTSCHEN REFERENZKORPUS}

\section{Einleitung}

Im vorliegenden Beitrag werden die Ergebnisse einer synchronischen Untersuchung zu deutschen Wortbildungskonstruktionen (im Weiteren WBK) auf -fritze, wie sie in den Beispielen 1-3 illustriert sind, vorgelegt.

(1) Die Werbefritzen nahmen nämlich nicht das Original, sondern ließen es von Kinderstimmen nachsingen und texteten es so um, dass der Reim nicht mehr funktionierte. (FOC04/SEP.00372 FOCUS, 20.09.2004, S. 196-98) ${ }^{1}$

(2) Mikrofonsalat, mehr als hundert ausländische Schreiberlinge und Fernsehfritzen drängeln sich im österreichischen Olympia-Haus. Alles nur wegen dieser Dopingsache. (FLT06/MAR.00090 Falter, 08.03.2006, S. 8)

(3) Ich bin hier hauptberuflich Bettelfritze, sagt der Ex-Banker, der ehrenamtlich den Düsseldorfer Karneval organisiert. (U17/OKT.01644 SZ, 13.10.2017, S. 8)

In den obigen Beispielen wird das Letztglied -fritze nicht als Eigenname, sondern als Gattungsname verwendet. Es handelt sich dabei um sog. deonymische Personenbezeichnungen, d. h. appellativische Substantive, die auf der Basis von Eigennamen gebildet worden sind. Sie bezeichnen dementsprechend nicht wie das freie lexikalische Morphem Fritz(e) Menschen mit demselben Namen, sondern (vorwiegend männliche) Personen mit irgendeinem gemeinsamen Merkmal.

Diese veränderte, abstraktere Bedeutung oder Entkonkretisierung, die sich aus der Wandlung vom Eigennamen zum Gattungsnamen ergibt, ist m. E. einer der wichtigsten Gründe dafür, das Letztglied -fritze als Suffixoid und die damit gebildeten WBK als Übergangsphänomene zwischen Komposition und Derivation zu klassifizieren. ${ }^{2}$ In diesem Beitrag werden sie formal als

1 Alle Beispiele sind, wenn nicht anders vermerkt, dem Deutschen Referenzkorpus entnommen.

2 In der Fachliteratur wurde mehrmals der Versuch unternommen, die Affixoide von den Affixen und den freien lexikalischen Morphemen abzugrenzen. Elsen (2009: 325 f.) hält z. B. außer der lexikalischen Bedeutung noch die Kriterien Gebundenheit, Positionsfestigkeit, automatische Reihenbildung, die Fähigkeit, sich mit Affixen direkt zu verbinden, das freie lexikalische Pendant, den lautlichen Gehalt und die Akzentuierbarkeit für relevant. 
Komposita behandelt und mit Methoden und Termini beschrieben, die bei der Modellierung von Zusammensetzungen üblich sind. ${ }^{3}$

\section{Darstellung der -fritze-Bildungen im Wörterbuch}

Es stellt sich zunächst die Frage, welche Informationen einsprachige deutsche Nachschlagewerke über -fritze-Bildungen enthalten. Unsere Stichprobenuntersuchung erstreckte sich auf die zwölfbändige Duden-Reihe, auf Langenscheidts Großwörterbuch Deutsch als Fremdsprache und das Digitale Wörterbuch der deutschen Sprache. Es konnte festgestellt werden, dass insgesamt 19 Wortbildungskonstruktionen auf -fritze lexikographisch kodifiziert sind, u. zw.:

Autofritze (DBW), Bummelfritze (DWDS) ${ }^{4}$, Fahrradfritze (Do) $)^{5}$, Filmfritze (DUW, LDaF, DWDS), Gemüsefritze (DWDS), Immobilienfritze (DUW, LDaF), Kleckerfritze (DUW), Mäkelfritze(Do), Meckerfritze(DUW, LDaF), Möbelfritze(DWDS), Nörgelfritze (LDaF), Quasselfritze (LDaF, DS)6, Quatschfritze (DWDS)7, Schuhfritze (DWDS), Trödelfritze (DR) ${ }^{8}$, Versicherungsfritze (LDaF), Werbefritze (DUW), Zeitungsfritze (DUW, LDaF) und Zigarrenfritze (DWDS).

Den untersuchten Wörterbüchern zufolge werden Bildungen auf -fritze nach dem schwachen Deklinationstyp flektiert (DUW, Do). Sie sind begrenzt produktiv (LDaF), gesprochen, pejorativ (LDaF), umgangssprachlich, abwertend (DUW, DBW) und kennzeichnen eine männliche Person, die

- sehr allgemein durch etwas charakterisiert ist (DUW, DWDS),

- beruflich mit etwas zu tun hat oder etwas oft tut (LDaF) und

- durch Beschäftigung mit etwas, den Verkauf von etwas charakterisiert ist (DBW).

Wie man sieht, wird unserem Untersuchungsgegenstand in den einsprachigen Print- und Online-Wörterbüchern relativ wenig Aufmerksamkeit gewidmet. Um stichhaltige Informationen über die -fritze-Bildungen zu bekommen, brauchen wir den realen Gebrauch widerspiegelnde Sprachdaten als Materialgrundlage, die ein repräsentatives Korpus bieten kann.

3 Vgl. dazu Eisenbergs Meinung (2013: 209): „In den meisten Fällen kann man die Wörter mit Halbaffix formal als Komposita behandeln....".

4 In unserem Korpus nicht belegt.

5 In unserem Korpus nicht belegt.

6 In unserem Korpus nicht belegt.

7 In unserem Korpus nicht belegt.

8 In unserem Korpus nicht belegt. 


\section{Ziel und Methode der Untersuchung}

Die zentralen Fragen der Untersuchung können in zwei Gruppen geteilt werden. Zunächst soll sich der Beitrag mit folgenden quantitativen Fragen auseinandersetzen:

- Wie viele verschiedene Lexeme können mit dem Letztglied -fritze in unseren Quellen belegt werden? (Typenzahl)

- Wie hoch ist ihre Verwendungsfrequenz? (Tokenzahl)

Nach der Beantwortung der obigen Fragen stehen die morphosyntaktischen Besonderheiten des Wortbildungsprodukts im Mittelpunkt, u. zw. folgende Kernpunkte:

- die Wortbildungsart und die Struktur der belegten WBK,

- die Wortklassenzugehörigkeit bzw. der formale Status der Konstituenten,

- die Anzahl,

- die hierarchische Struktur und

- die Wortbildungsart der Kompositionsglieder.

Die oben skizzierten Forschungsfragen sollen anhand von authentischem Sprachmaterial und mit Hilfe von korpuslinguistischen Methoden beantwortet werden. Als Datenbasis dient dazu das Deutsche Referenzkorpus (DeReKo) des Leibniz-Instituts für Deutsche Sprache Mannheim. Dieses enthält u. a. belletristische, wissenschaftliche und populärwissenschaftliche Texte sowie Zeitungstexte in einem Umfang von 46,9 Milliarden Wörtern. ${ }^{9}$

\section{Methode und Ergebnisse der Korpusuntersuchung}

\subsection{Typen- und Tokenzahl}

Die der Korpusuntersuchung zugrunde gelegte Suchanfrage war *fritze+, wobei der erste Platzhalteroperator $\left(^{*}\right)$ für null bis beliebig viele, der zweite (+) für höchstens ein Zeichen steht. Mit Hilfe der Kombinationssuche konnten im Archiv der geschriebenen Sprache alle Zeichenketten mit dem Letztglied -fritze oder -fritzen ermittelt werden. Die erste, temporäre Wortformliste enthielt 840 Wortformen. Fehltreffer wie Vor- und Familiennamen (z. B. Fritze oder Fritzer), Ableitungen von Ortsnamen (Molfritzer) und sonstige falsche Treffer (Hufritze, aufritzen usw.) wurden manuell entfernt.

9 Stand 18.01.2020. Vgl. https://www1.ids-mannheim.de/kl/projekte/korpora/. 
Die endgültige Liste enthält 509 Types und 1914 Tokens. Tab. 1 zeigt die Belege mit der größten Vorkommenshäufigkeit.

\begin{tabular}{|c|l|c|}
\hline 1. & Werbefritze & 245 \\
\hline 2. & Pressefritze & 114 \\
\hline 3. & Fernsehfritze & 89 \\
\hline 4. & Meckerfritze & 88 \\
\hline 5. & Zeitungsfritze & 88 \\
\hline 6. & Radiofritze & 61 \\
\hline 7. & Filmfritze & 54 \\
\hline 8. & Marketing-Fritze & 46 \\
\hline 9. & Reklamefritze & 36 \\
\hline 10. & Computerfritze & 27 \\
\hline 11. & Versicherungsfritze & 26 \\
\hline 12. & Medienfritze & 25 \\
\hline 13. & Öko-Fritze & 23 \\
\hline 14. & Immobilienfritze & 22 \\
\hline 15. & PR-Fritze & 19 \\
\hline 16. & Feuerfritze & 17 \\
\hline 17. & Nörgelfritze & 17 \\
\hline 18. & Fahrradfritze & 16 \\
\hline 19. & Modefritze & 16 \\
\hline 20. & Plapperfritze & 13 \\
\hline & & \\
\hline
\end{tabular}

Tab. 1: Belege mit der größten Vorkommenshäufigkeit. Fett gedruckt: Lexikographisch kodifizierte Lexeme.

Vergleicht man die obige Liste mit der der lexikographisch kodifizierten -fritze-Bildungen, wird deutlich, dass nur $40 \%$ der in den untersuchten Wörterbüchern lemmatisierten WBK auf -fritze es geschafft haben, unter die 20 häufigsten Types unserer Korpusuntersuchung zu kommen. Deswegen liegt die Vermutung nahe, dass in den einsprachigen Wörterbüchern nicht unbedingt diejenigen WBK auf -fritze Eingang gefunden haben, die in der Schreibpraxis die größte Textfrequenz aufweisen. den:

Betrachtet man die Belegzahlen genauer, kann Folgendes festgehalten wer-

Das Zweitglied -fritze weist Reihenbildung auf. Die Typenzahl kann mit 509 verschiedenen Lexemen als hoch gewertet werden. Dies ist ein mögliches Indiz für eine hohe Produktivität der untersuchten WBK, denn je produktiver ein Wortbildungsmodell ist, desto mehr Wörter können damit gebildet werden (vgl. Schneider-Wiejowski 2011: 94). 
Im Korpus finden sich zahlreiche usuelle Wörter (Meckerfritze, Nörgelfritze, Versicherungsfritze, Zeitungsfritze usw.), aber das Wortbildungsmodell dient auch als Muster für Neubildungen. Die Zahl der Einzelbelege ist hoch (329), sie erreichen einen Anteil von mehr als $64 \%$ aller Bildungen. Unter ihnen ist die Frequenz der Gelegenheitsbildungen besonders hoch, vgl. z. B. Anzug-und-Krawatte-Fritze ,jemand, der oft Anzug und Krawatte trägt', Grünkramfritze ,Gemüseverkäufer', Law-andorder-Fritze (sic!) ,eine Person, die die allgemeinverbindlichen Regeln einhält', Pommesbuden-Laberfritze ,Schwätzer an einem Imbissstand', Schickimicki-Fritze ,Modenarr', Technischer-Überwachungs-Fritze ,Mitarbeiter des Technischen Überwachungs-Vereins', Upperclass-Fritze ,Angehöriger der Oberschicht', Witzhemdchenfritze ,eine Person, die witzige Hemden trägt' oder ZDF-Frühstücksfernsehfritze ,Mitarbeiter des ZDF-Frühstücksfernsehens'.

Bezüglich der Einzelbelege verdient die These von Fleischer/Barz (2012: 75) Aufmerksamkeit: „Enthält ein umfangreiches Textkorpus eine große Anzahl von Hapaxlegomena eines Modells [...], zeugt dieser Befund von hoher Produktivität des Modells."

Andererseits weisen nur wenige Lexeme eine besonders hohe Textfrequenz auf: Nur 28 Types (5,5\%) haben eine Vorkommenshäufigkeit von zehn oder mehr Belegen. Dies spricht gegen eine hohe Produktivität des Wortbildungsmodells (vgl. Fleischer/Barz 2012: 75).

\subsection{Die Wortbildungsart}

Wie oben erwähnt, werden Bildungen auf -fritze in diesem Beitrag als Komposita behandelt. Innerhalb dieser gehören sie zur Gruppe der sog. Determinativkomposita, weil zwischen den unmittelbaren Konstituenten (im Weiteren: UK) Subordinationsbeziehung herrscht.

Die untersuchten WBK sind binär gegliedert: Die erste unmittelbare Konstituente (A-Konstituente oder Erstglied) ist das Bestimmungswort, die zweite (B-Konstituente oder Zweitglied) fungiert als Grundwort und bestimmt die grammatischen Merkmale sowie die lexikalische Bedeutung des Kompositums..$^{10}$

\subsection{Wortklassenzugehörigkeit bzw. formaler Status der Konstituenten}

Das Grundwort der B-Konstituente ist in unserem Fall jeweils durch ein Substantiv (nämlich -fritze) repräsentiert, wir haben es demnach jeweils mit Substantivkomposita zu tun.

Wie Tab. 2 zeigt, erscheinen im Korpus substantivische Erstglieder mit hoher Frequenz, die Anteile der übrigen A-Konstituenten (Verb, Adjektiv, Konfix,

10 Vgl. dazu: Donalies (52 f.). 
Phrase bzw. Satz) bleiben unter $9 \%$.

\begin{tabular}{|l|c|c|}
\hline $\begin{array}{c}\text { Wortklassenzugehörigkeit } \\
\text { bzw. formaler Status der } \\
\text { A-Konstituente }\end{array}$ & Absolute Häufigkeit & Relative Häufigkeit \\
\hline Substantiv & 428 & $84,09 \%$ \\
\hline Verb & 44 & $8,64 \%$ \\
\hline Adjektiv & 5 & $0,98 \%$ \\
\hline Konfix & 9 & $1,77 \%$ \\
\hline Phrase/Satz & 23 & $4,52 \%$ \\
\hline Gesamt & 509 & $100 \%$ \\
\hline
\end{tabular}

Tab. 2: Wortklassenzugehörigkeit bzw. formaler Status der A-Konstituente

Die Feststellung der Wortklassenzugehörigkeit der Erstglieder stößt in vielen Fällen auf Probleme, weil sich formal mehrere Interpretationsmöglichkeiten ergeben, vgl. Angelfritze, Baufritze, Jammerfritze, Jodelfritze, Jubelfritze, Rummelfritze und Zauberfritze. Das Bestimmungswort dieser Bildungen kann sowohl als Substantiv als auch als Verbstamm aufgefasst werden. ${ }^{11}$ Eine mögliche Lösung für das Problem bietet die Paraphrasierung wie z. B.: ,ein Angelfritze ist eine Person, die oft oder gern angelt'. Fazit: verbales Erstglied.

Wenn dagegen nicht nur formale, sondern auch semantische Doppelmotivation vorliegt, hilft die Paraphrasierung auch nicht weiter, vgl. ,ein Rummelfritze ist eine Person, die oft rummelt/Rummel macht'. In diesem Fall bleibt es der subjektiven Einschätzung des Verfassers überlassen, sich für die eine oder andere Interpretation zu entscheiden.

\subsubsection{Erstglied Substantiv}

\section{Anzahl der Kompositionsglieder}

Im Korpus finden sich insgesamt 319 zwei-, 100 drei- und 9 viergliedrige Komposita mit substantivischer A-Konstituente.

11 Zur Berührung von substantivischem und verbalem Erstglied vgl. Fleischer/Barz (1995: 109). 


\section{Zweigliedrige Komposita}

\section{Wortbildungsart ${ }^{12}$}

Betrachtet man die A-Konstituenten der zweigliedrigen Komposita näher, wird deutlich, dass die Erstglieder in den meisten Korpusbeispielen durch freie Grundmorpheme (z. B. Bierfritze, Dorffritze, Müllfritze) repräsentiert sind (vgl. Tab. 3).

\begin{tabular}{|c|c|c|c|}
\hline \multicolumn{3}{|c|}{ Freie Grundmorpheme (Simplexe) } & 153 \\
\hline \multirow{5}{*}{ Explizite Derivate } & \multicolumn{2}{|l|}{ Suffixderivate } & 76 \\
\hline & \multicolumn{2}{|l|}{ Präfixderivate } & 7 \\
\hline & \multicolumn{2}{|l|}{ Präfix-Suffix-Derivate } & 6 \\
\hline & \multicolumn{2}{|c|}{ Kombinatorische Derivate } & 10 \\
\hline & \multicolumn{2}{|l|}{ Konfixderivate } & 2 \\
\hline \multicolumn{3}{|l|}{ Implizite Derivate } & 4 \\
\hline \multicolumn{3}{|l|}{ Konvertate } & 3 \\
\hline \multicolumn{3}{|l|}{ Rückbildungen } & 2 \\
\hline \multirow{5}{*}{ Kurzwörter } & \multirow{3}{*}{ Multisegmentale KW } & Buchstabenkurzwörter & 27 \\
\hline & & Silbenkurzwörter & 4 \\
\hline & & Mischkurzwörter & 4 \\
\hline & \multirow{2}{*}{ Unisegmentale KW } & Kopfwörter & 19 \\
\hline & & Schwanzwörter & 2 \\
\hline \multicolumn{3}{|l|}{ Gesamt } & 319 \\
\hline
\end{tabular}

Tab. 3: Wortbildungsarten der substantivischen Erstglieder der zweigliedrigen Komposita

$\mathrm{Zu}$ den häufigsten Wortbildungsarten gehören explizite Derivation und Kurzwortbildung (vgl. Tab. 3).

Innerhalb der expliziten Derivation spielt die Suffixderivation (z. B. Agenturfritze, Bildungsfritze, Gesellschaftsfritze) eine führende Rolle, während Präfixderivate (z. B. Bezirksfritze, Gehirnfritze, Getränkefritze, Immobilienfritze, Reformfritze, Reklamefritze), Präfix-Suffix-Derivate (z. B. Entscheidungsfritze, Unterhaltungsfritze, Verbesserungsfritze, Verbindungsfritze, Versicherungsfritze), kombinatorische Derivate (Gemeindefritze, Gemüsefritze, Gewerkschaftsfritze, Veranstaltungsfritze, Vergasungsfritze, Vermarktungsfritze, Verschwörungsfritze, Versorgungsfritze, Verteidigungsfritze, Verwaltungsfritze) und Konfixderivate (Investmentfritze, Programmfritze) seltener in Erscheinung treten.

12 Bei der Bestimmung der jeweiligen Wortbildungsart der Kompositionsglieder waren wir bestrebt, so weit wie möglich auch diachronische Aspekte zu berücksichtigen. 
Unter den Kurzwörtern dominieren die Buchstabenkurzwörter. Diese bezeichnen vorwiegend:

- $\quad$ politische Vereinigungen bzw. politische Ideologien wie AfD-Fritze $(<A /$ ternative für Deutschland), CDU-Fritze (<Christlich-Demokratische Union), EP-Fritze (< Ethnopluralismus), FDP-Fritze (< Freie Demokratische Partei [Deutschlands]), KB-Fritze (<Kommunistischer Bund), NPD-Fritze (< National-Demokratische Partei Deutschlands), NS-Fritze (< Nationalsozialist), Pegida-Fritze (< Patriotische Europäer gegen [die] Islamisierung des Abendlandes) und SED-Fritze (< Sozialistische Einheitspartei Deutschlands);

- $\quad$ staatliche oder zwischenstaatliche Organisationen wie BfN-Fritze (< Bundesamt für Naturschutz), CIA-Fritze (< Central Intelligence Agency), EU-Fritze (< Europäische Union), UNMIK-Fritze (< United Nations Interim Administration Mission in Kosovo) und UNO-Fritze ( $<$ United Nations Organization);

- Sportverbände wie FIFA-Fritze (< Fédération Internationale de Football Association) und Uefa-Fritze (sic!) (< Union of European Football Associations);

- Wirtschaftszweige wie IT-Fritze (< information technology), ÖV-Fritze (< Öffentlicher Verkehr), PR-Fritze (< Public Relations) und SD-Fritze (< Sicherheitsdienst);

- Namen von Firmen wie BMW-Fritze (< Bayerische Motoren Werke) und CS-Fritze (< Credit Suisse);

- Sonstiges wie Kfz-Fritze (< Kraftfahrzeug), SUV-Fritze (< sport utility vehicle), TV-Fritze (< Television) und UFO-Fritze (< unidentified flying object).

Andere multisegmentale Kurzwörter wie Silbenkurzwörter (Kripo-Fritze < Kriminalpolizei, Moped-Fritze < Motorveloziped/Motor u. Pedal, Stasifritze < Staatssicherheitsdienst, Toto-Fritze $<$ Totalisator $^{13}$ ) und Mischkurzwörter (AstaFritze < Allgemeiner Studentenausschuss, Flakfritze < Fliegerabwehrkanone, Kinofritze < Kinematograph) finden sich im Korpus nur selten.

Was die unisegmentalen Kurzwörter anbelangt, kann von einer eindeutigen Dominanz der Kopfwörter gesprochen werden: Autofritze $(<$ Automobil), Comicfritze (<Comicstrip), Country-Fritze (<Countrymusic), Diskofritze (<Diskothek), Expo-Fritze (< Exposition), Fotofritze (< Fotografie), Infofritze (< Information), Mathefritze (< Mathematik), Nazi-Fritze (< Nationalsozialist), Plagifritze (< Plagium), Pop-Fritze (< Popmusik), Porno-Fritze (< Pornographie), Radiofritze (< engl. radiotelegraphy), Rockfritze (< Rockmusik), Techno-Fritze (< engl. technological) und ZooFritze (< zoologischer Garten).

Bei T-Fritze ,Mitarbeiter der Firma Telekom' erscheint ein einzelner Buchstabe als Erstglied.

13 Staatliche Einrichtung zum Abschluss von Wetten auf Rennpferde (DUW). 
In den Erstgliedern der Komposita Wessi-Fritze (< Westdeutscher) und Schoggifritze ,Schweizer Schokoladesorte' (< Schokolade) finden Reduktion und Derivation mit dem Suffix -i gleichzeitig statt.

Die Zahl der Schwanzwörter wie Chemiefritze (<Alchemie) und Plattenfritze $(<$ Schallplatte) ist niedrig.

Zu den seltenen Wortbildungsarten im Korpus gehören des Weiteren implizite Derivation (Kunstfritze, Schlossfritze, Verbandsfritze, Verlagsfritze), Rückbildung (Knallfritze, Vereinsfritze) und Konversion. Basis zum Konversionsprodukt liefern in zwei Fällen Verbstämme (Baufritze und Verkehrsfritze), in einem Fall eine Partizip-II-Form (Beamtenfritze).

\section{Dreigliedrige Komposita}

\section{Hierarchische Struktur der Kompositionsglieder}

Betrachtet man die Determinationsrelation der Konstituenten, ist die überwiegende Mehrheit der dreigliedrigen Komposita (97 von 100 Types) unserer Korpusanalyse zufolge linksverzweigt, z. B. Handbuchfritze, Landwirtschaftsfritze und Radsportfritze. Rechtsverzweigung ist nur in drei Fällen belegt, u. ZW. FDPWirtschaftsfritze, OECD-Bildungsfritze und TV-Versicherungsfritze.

Wortklassenzugehörigkeit bzw. formaler Status der Kompositionsglieder

\begin{tabular}{|l|l|l|l|}
\hline A-Konstituente & B-Konstituente & $\begin{array}{l}\text { Absolute } \\
\text { und relative } \\
\text { Häufigkeit }\end{array}$ & Belege \\
\hline Subst. + Subst. & Subst. & $58(58 \%)$ & $\begin{array}{l}\text { Z. B. Barkassenfritze, Handbuchfritze, } \\
\text { Landwirtschaftsfritze, } \\
\text { Schriftstellerfritze }\end{array}$ \\
\hline Präp. + Subst. & Subst. & $10(10 \%)$ & $\begin{array}{l}\text { Andenkenfritze, Anlagenfritze, } \\
\text { Antriebsfritze, Anzeigenfritze, } \\
\text { Anzugfritze, Aufsichtsfritze, } \\
\text { Ausbildungsfritze, Ausdauerfritze, } \\
\text { Umfrage-Fritze, Umweltfritze }\end{array}$ \\
\hline Verb + Subst. & Subst. & $10(10 \%)$ & $\begin{array}{l}\text { Drehbuchfritze, Fahrradfritze, } \\
\text { Mietwagenfritze, Nähmaschinenfritze, } \\
\text { Parkplatzfritze, Schallplattenfritze, } \\
\text { Sparkassen-Fritze, Talkshowfritze, } \\
\text { Waschmaschinenfritze, Wettbürofritze }\end{array}$ \\
\hline Adj. + Subst. & Subst. & $7(7 \%)$ & $\begin{array}{l}\text { Biedermeierfritze, Fremdenverkehrs- } \\
\text { Fritze, Greenpeace-Fritze, } \\
\text { Grünkramfritze, Hardcore-Fritze, } \\
\text { Schickimicki-Fritze, Upperclass-Fritze }\end{array}$ \\
\hline Konfix + Subst. & Subst. & $4(\%)$ & $\begin{array}{l}\text { Multikulti-Fritze, Telefonfritze, } \\
\text { Monokelfritze, Paläo-Diät-Fritze }\end{array}$ \\
\hline
\end{tabular}




\begin{tabular}{|l|l|l|l|}
\hline Verb + Konfix & Subst. & $1(1 \%)$ & Tankwartsfritze \\
\hline Part. II + Subst. & Subst. & $2(2 \%)$ & $\begin{array}{l}\text { Gebrauchtwagenfritze, } \\
\text { Gemischtwarenfritze }\end{array}$ \\
\hline Pron. + Subst. & Subst. & $1(1 \%)$ & Selbstdarstellungsfritze \\
\hline Numer. +Subst. & Subst. & $1(1 \%)$ & Zweiradfritze \\
\hline Adv. + Subst. & Subst. & $1(1 \%)$ & Außenhandelsfritze \\
\hline Subst. + Konfix & Subst. & $1(1 \%)$ & Bürokratenfritze \\
\hline Konfix + Konfix & Subst. & $1(1 \%)$ & Automatenfritze \\
\hline Subst. & Subst. + Subst. & $3(3 \%)$ & $\begin{array}{l}\text { TV-Versicherungsfritze, FDP- } \\
\text { Wirtschaftsfritze, OECD- } \\
\text { Bildungsfritze }\end{array}$ \\
\hline Gesamt: & & $100(100 \%)$ & \\
\hline
\end{tabular}

Tab. 4: Wortklassenzugehörigkeit bzw. formaler Status der Kompositionsglieder in den dreigliedrigen Komposita

Die Mehrheit der A-Konstituenten der dreigliedrigen Zusammensetzungen machen mit 58 \% Substantiv-Substantiv-Komposita aus (vgl. Tabelle 4). Mit gewisser Häufigkeit sind noch Präposition-Substantiv- (10 \%), Verb-Substantiv(10 \%) und Adjektiv-Substantiv-Komposita (7 \%) zu finden.

Als Erstglied der A-Konstituenten können außerdem Konfixe, Verben, Partizipien, Pronomen, Numeralien oder Adverbien erscheinen, ihr Anteil ist jedoch niedrig. Schickimicki-Fritze ist eine sprachspielerische Bildung zum Adjektiv schick.

Konfixe können mit anderen Konfixen, Substantiven oder Verbstämmen Komposita bilden.

In drei Fällen erscheint die B-Konstituente in Form von Zusammensetzungen, die durchgehend aus substantivischen Kompositionsgliedern bestehen. ${ }^{14}$

14 Nach Ortner (1991: 16) ist das Bestimmungswort der dreigliedrigen Substantivkomposita „fast ausschließlich (zu 98 \%) aus Wörtern der Hauptwortarten Substantiv, Verb und Adjektiv (mit Adverbien und Numeralia) gebildet; - in welcher Kombination, das ergibt sich vor allem aus der kommunikativen Funktion des Determinativkompositums, der (Bekanntheit und der entsprechenden) Gebräuchlichkeit (und "semantischen Verträglichkeit“) der Lexeme und ihren - wortartbedingten - Kombinationsmöglichkeiten. Von daher zeigt sich, daß die größte Produktivität bei den morphologischen Subtypen liegt, deren ,Mittelstück' ein Substantiv ist ...". 


\section{Wortbildungsart}

Die A-Konstituenten der dreigliedrigen Komposita sind in zahlreichen Fällen aus freien Grundmorphemen zusammengesetzt, z. B. Handbuchfritze, Radsportfritze, Landkreisfritze. Gelegentlich erscheinen die ersten UK als partielles Kurzwort (E-Roller-Fritze < Elektromotorroller, TdS-Felgenfritze < TurbodieselSport-Felgen), Buchstabenkurzwort (FDP-Wirtschaftsfritze < Freie Demokratische Partei, OECD-Bildungsfritze < Organization for Economic Cooperation Development, TV-Versicherungsfritze) Reduplikationsform (Schickimicki-Fritze ,modischer Tand'), ${ }^{15}$ Rückbildung (Zweiradfritze < zweirädrig) oder Klammerform (Bierdeckel-Fritze < Bierglasdeckel). Letzteres Beispiel kann als Übergangsform zu einem viergliedrigen Kompositum angesehen werden.

Durch Wortkreuzung sind die A-Konstituenten folgender dreigliedriger Komposita entstanden: Infotainment-Fritze (< Information + Entertainment), Internetfritze ,Mitarbeiter des Internetanbieters' $(<\text { international + network })^{16}$ und Randalefritze (vom mundartlichen obd., omd. Rand, Rant ,Possen, lärmender Spaß, Tumult' + Skandal, vgl. DWDS).

Das Bestimmungswort der A-Konstituente kann als Ergebnis einer Suffixderivation (Finanzkrisen-Fritze, Stöcklschuhfritze, Urheberrechtsfritze), einer Präfixderivation (Getränkemarktfritze), einer impliziten Derivation (z. B. Flughafenfritze, Flugsicherungs-Fritze, Schriftstellerfritze) oder einer Konversion (z. B. Lebensberatungsfritze, Rechenzentrums-Fritze, ${ }_{17}^{17}$ Baumarkt-Fritze) vorliegen.

Das Grundwort der A-Konstituente kann über ein Simplex hinaus ein Suffixderivat (Landwirtschaftsfritze, Tageszeitungsfritze, Ferienwohnungsfritze), ein implizites Derivat (Naturschutzfritze, Staatsschutzfritze), ein kombinatorisches Derivat (Datenverarbeitungsfritze, Lebensberatungsfritze), ein Präfix-Suffix-Derivat (Selbstdarstellungsfritze) oder ein Rückbildungsprodukt (Knallfritze, Vereinsfritze) sein.

Das Grundwort der B-Konstituente ist in jedem Fall ein freies Grundmorphem (Fritze), das Bestimmungswort der zweiten UK erscheint dagegen als Suffixderivat (FDP-Wirtschaftsfritze, OECD-Bildungsfritze) oder als Präfix-SuffixDerivat (TV-Versicherungsfritze).

\section{Viergliedrige Komposita}

Ihre Zahl ist zwar niedrig, aber im Korpus kommen auch lexikographisch nicht kodifizierte, aus vier Grundmorphemen bestehende polymorphemische substantivische Komposita vor.

15 Fleischer/Barz (1995: 48) sprechen in diesem Fall von Reimdoppelung.

16 Herberg/Kinne/Steffens (2004: 175).

17 Der Verbstamm rechnen erscheint in Zusammensetzungen in der Form Rechen-. 


\section{Innere Struktur der Komposita}

Die Anteile der verschiedenen Verzweigungstypen gestalten sich folgendermaßen:

Linksverzweigt sind die folgenden sieben Komposita: Filmförderungskommissionsfritze, Fußball-Verbands-Fritze, Kabelfernsehfritze, KaltwarenMarketingfritze, Landwirtschaftskammer-Fritze, SF-Fernsehfritze und TrolleybusMagazine-Fritze (vgl. Grafik 1).

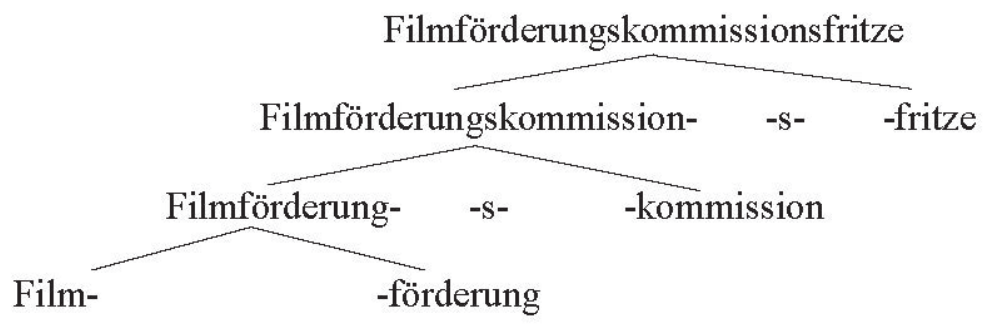

Grafik 1: Beispiel für Linksverzweigung: Filmförderungskommissionsfritze

Eine symmetrische Links-Rechts-Verzweigung weisen die Komposita AbsatzOberfritze und Pommesbuden-Laberfritze auf (vgl. Grafik 2).

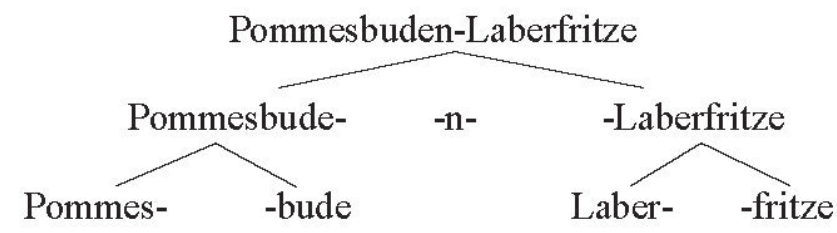

Grafik 2: Beispiel für Lins-Rechts-Verzweigung: Pommesbuden-Laberfritze 


\section{Wortklassenzugehörigkeit der Kompositionsglieder}

\begin{tabular}{|l|l|l|}
\hline A-Konstituente & B-Konstituente & Belege \\
\hline & & $\begin{array}{l}\text { Filmförderungskommissionsfritze, } \\
\text { Fußball-Verbands-Fritze, } \\
\text { Subst. + Subst. + Subst. }\end{array}$ \\
& Subst. & $\begin{array}{l}\text { Kabelfernsehfritze, } \\
\text { Landwirtschaftskammer-Fritze, } \\
\text { SF-Fernsehfritze, Trolleybus-Magazine- } \\
\end{array}$ \\
& & Fritze \\
\hline Adj. + Subst. + Subst. & Subst. & Kaltwaren-Marketingfritze \\
\hline Subst. + Subst. & Verbstamm + Subst. & Pommesbuden-Laberfritze \\
\hline Präp. + Subst. & Adj. + Subst. & Absatz-Oberfritze \\
\hline
\end{tabular}

Tab. 5: Wortklassenzugehörigkeit der Kompositionsglieder der viergliedrigen Komposita

Die A-Konstituenten der linksverzweigten viergliedrigen Komposita bestehen bis auf eine Ausnahme aus substantivischen Kompositionsgliedern.

In den links-rechts-verzweigten Komposita erscheinen neben den substantivischen Grundwörtern substantivische, adjektivische, präpositionale oder verbale Konstituenten (vgl. Tab. 5).

\section{Wortbildungsart}

An der Bildung der A-Konstituenten können Simplizia (z. B. Landwirtschaftskammer-Fritze), Suffixderivate (Filmförderungskommissionsfritze), implizite Derivate (Absatz-Oberfritze, Fußball-Verbands-Fritze), Konversionsprodukte (Kabelfernsehfritze [Fernsehen < fernsehen]) oder Kurzwörter (SF-Fernsehfritze > Science-Fiction) beteiligt sein.

Die komplexen B-Konstituenten setzen sich ausschließlich aus simplizischen Kompositionsgliedern zusammen.

\subsubsection{Erstglied Verb}

Wortbildungskonstruktionen mit verbalen A-Konstituenten bilden mit einer absoluten Häufigkeit von 44 Types die zweitgrößte Gruppe im Korpus. Zweigliedrige Zusammensetzungen stellen mit 37 Lexemen den Grundtyp dar, überdies finden sich fünf dreigliedrige (z. B. Vodafone-Werbefritze) und zwei viergliedrige Komposita(Geschenkpapier-Bastelfritze und Musikwirtschaftswerbefritze) im Datenmaterial.

$18, \ldots . .$. in Komposita mit Fern- [liegt] vielfach nicht das Adjektiv, sondern das Substantiv die Ferne vor ..." (Fleischer/Barz 1995: 104). 
Unter den Erstgliedern gibt es zahlreiche Verbstämme, denen Verben auf -(e)In und -(e)rn zugrunde liegen. Sie sind entweder Simplexe wie Angelfritze, Babbelfritze, Plauderfritze, Rummelfritze oder Suffixderivate. Letztere sind herkunftsmäßig Iterativbildungen (z. B. Bettelfritze ${ }^{19}$, Drängelfritze ${ }^{20}$, Kleckerfritze $^{21}$, Kletterfritze ${ }^{22}$, Laberfritze ${ }^{23}$, Mäkelfritze ${ }^{24}$, Meckerfritze ${ }^{25}$, Plapperfritze ${ }^{26}$, Sabbelfritze ${ }^{27}$, Strampelfritze ${ }^{28}$, Wanderfritze ${ }^{29}$, Zündelfritze $\left.{ }^{30}\right)$, oder Intensivbildungen (Rubbelfritze ${ }^{31}$, Stotterfritze ${ }^{32}$ ), vereinzelt Diminutiv- (Lächelfritze ${ }^{33}$ ) oder Faktitivbildungen (Sammelfritze ${ }^{34}$ ).

Das Erstglied von Jodelfritze ist vom Jodelruf jo abgeleitet (vgl. DHW).

Die A-Konstituenten von Jammerfritze, Schmierfritze und Zauberfritze sind Konversionsprodukte.

In einigen Fällen wie Heulfritze, Schwafelfritze, Wuselfritze und Zappelfritze kann die Herkunft der ersten UK nicht sicher geklärt werden (vgl. Tab 6).

\begin{tabular}{|l|c|}
\hline Freie Grundmorpheme (Simplexe) & 9 \\
\hline Suffixderivate & 21 \\
\hline Konvertate & 3 \\
\hline Herkunft ist nicht sicher geklärt & 4 \\
\hline Gesamt & $\mathbf{3 7}$ \\
\hline
\end{tabular}

Tab. 6: Wortbildungsarten des verbalen Erstglieds der zweigliedrigen Komposita

19 Iterativbildung zu bitten (vgl. Pfeifer 1993).

20 Iterativbildung zu drängen vgl. Pfeifer 1993).

21 Iterativbildung zu klecken (vgl. DHW).

22 Iterativbildung zu frühnhd. kletten (vgl. Pfeifer 1993).

23 Iterativbildung zu mnd., nd. labben, schlürfend trinken, lecken, schlecken' (vgl. Pfeifer 1993).

24 Iterativbildung zu nl. maken ,machen, handeln' (vgl. DHW).

25 Iterativbildung zu schallnachahmendem mecken (vgl. DHW).

26 Iterativbildung zu blappen, plappen (vgl. DW).

27 Iterativbildung zu mnd. sabben (vgl. Pfeifer 1993).

28 Iterativbildung zu mnd. strampen ,mit den Füßen hart auftreten, aufstampfen‘ (vgl. Pfeifer 1993).

29 Iterativbildung zu ahd. wantōn ,wenden, verwandeln, sich ändern' (vgl. Pfeifer 1993).

30 Iterativbildung zu zünden.

31 Intensivbildung zu reiben (vgl. Pfeifer 1993).

32 Intensivbildung zu stoßen (vgl. Pfeifer 1993).

33 Diminutivbildung zu lachen.

34 Faktitivbildung zu den Adverbien ahd. saman, mhd. samen(e), got. samana ,zusammen, zugleich, miteinander' (vgl. Pfeifer 1993). 
Die innere Struktur aller Komposita mit komplexem verbalem Erstglied ist durch Linksverzweigung gekennzeichnet (vgl. Grafik 3).

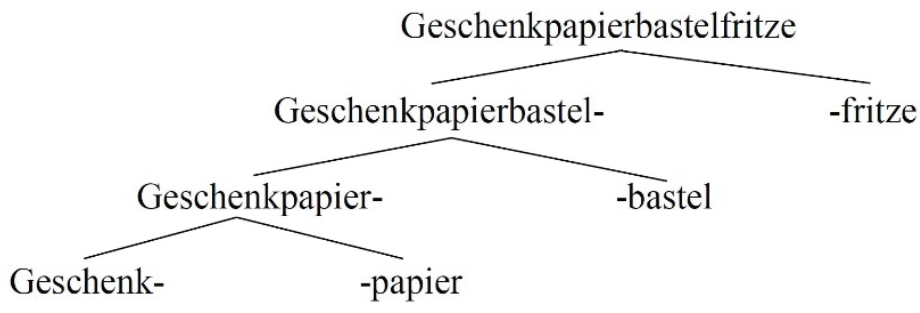

Grafik 3: Beispiel für Linksverzweigung: Geschenkpapier-Bastelfritze

In den A-Konstituenten können außer dem verbalen Grundwort Simplexe (Europa-Werbefritze), Buchstabenkurzwörter (FPÖ-Werbefritze, GPS-Werbefritze, TV-Werbefritze), Präfixderivate (Geschenkpapier-Bastelfritze) und Suffixderivate (Musikwirtschaftswerbefritze) vorkommen.

Das Grundwort des Erstglieds ist in allen Belegen mit Substantiven gekoppelt.

\subsubsection{Erstglied Adjektiv}

Alle fünfim Korpusvorkommenden Komposita mit adjektivischer A-Konstituente sind zweigliedrig strukturiert.

Simplizische, einsilbige Adjektive liegen in Grün-Fritze und Schlaufritze, ein Präfixderivat in Express-Fritze (i. S. v. ,Eilbote') und ein Suffixderivat in Kommunalfritze vor. Auch das Erstglied von Billigfritze ist aus diachronischer Sicht als Ergebnis einer Suffixderivation zu betrachten (vgl. DBW).

\subsubsection{Erstglied Konfix}

Die nur als gebundene Morpheme vorkommenden Konfixe dienen nicht nur als Derivationsbasen, sondern auch als Kompositionsglieder. Als Erstglieder beteiligen sie sich in unserem Korpus in neun Fällen an der Bildung zweigliedriger Komposita, nämlich Astrofritze, Biofritze, Elektrofritze, Öko-Fritze, Paläofritze, Pharma-Fritze, Politfritze, Psycho-Fritze und Telefritze. In allen diesen WBK handelt es sich um entlehnte Kompositionsglieder. 


\subsubsection{Erstglied Phrase/Satz}

Phrasen bzw. Sätze als erste UK machen im Korpus mit 23 Lexemen 4,52 \% aller Types aus.

Am häufigsten bestehen die Erstglieder aus Substantiven mit vorangestelltem adjektivischem Attribut, z. B. Halbstadtfritze ,Westberliner', New-Age-Fritze und Public-Relations-Fritze. Sie weisen subordinative Struktur auf. Die Flexion des Adjektivs wird in manchen Fällen beibehalten, vgl. Deutsche-Welle-Fritze und Technischer-Überwachungs-Fritze.

Als übliche koordinative Struktur kann die Reihung von zwei Substantiven im Erstglied belegt werden (z. B. Lotto-Toto-Fritze, Cocacola-Fritze [sic!], FengShui-Fritze [,Wind und Wasser']), wobei die Substantive auch explizit verbunden sein können (vgl. Anzug-und-Krawatte-Fritze, Law-andorder-Fritze [sic!], Post-\&Telekom-Werbefritze).

Seltener erscheinen komplexere polymorphemische Konstruktionen als erste UK wie Wald- und Wiesen-Pressefritze (sic!) und ZDF-Frühstücksfernsehfritze (,Mitarbeiter des ZDF-Frühstückfernsehens').

Die Personenbezeichnung Störenfritze in der Bedeutung ,Ruhestörer, Unruhestifter' ist eine sprachspielerische Wortbildung nach dem Muster von Störenfried. In der Fachliteratur werden derartige Wortbildungskonstruktionen als Satznamen (vgl. Fleischer/Barz 1995: 213), imperativische Satznamen (Henzen 1965: 83 f.) oder als Konversion von Imperativsätzen (vgl. Fleischer/ Barz 2012: 65) bezeichnet.

\section{Zusammenfassung und Ausblick}

Die Ergebnisse der Untersuchung lassen sich wie folgt zusammenfassen.

Unser Ausgangspunkt war die Feststellung, dass WBK auf -fritze, die im vorliegenden Beitrag als Determinativkomposita behandelt werden, in den gedruckten und Onlinewörterbüchern unterrepräsentiert sind. In den untersuchten einsprachigen Nachschlagewerken sind außerdem nicht immer die häufigsten Wortbildungen lemmatisiert.

Unsere synchronische, quantitative Korpusuntersuchung ergab, dass das Letztglied -fritze reihenbildend auftritt: In den Quellen konnten 509 Types und 1914 Tokens belegt werden.

Kompositionsaktiv verhält sich das Letztglied -fritze mit substantivischen Erstgliedern. Andere A-Konstituenten wie Verben, Konfixe, Adjektive, Phrasen bzw. Sätze finden sich dagegen viel seltener.

$77,2 \%$ der untersuchten Determinativkomposita sind zweigliedrig, $20,8 \%$ dreigliedrig und $2 \%$ viergliedrig aufgebaut. Drei- und viergliedrige Zusammensetzungen kommen in unserem Korpus nur bei substantivischen und verbalen Erstgliedern vor. 
Die Analyse der hierarchischen Struktur der mehrgliedrigen Komposita führte zu folgendem Ergebnis: Komplexere Konstruktionen konnten vor allem bei substantivischem - seltener bei verbalem - Erstglied belegt werden. Linksverzweigung liegt bei 97,2 \% der dreigliedrigen und 81,8 \% der viergliedrigen Komposita vor. Rechtsverzweigt sind 2,8 \% der dreigliedrigen, beidseitig verzweigt 18,2\% der viergliedrigen Zusammensetzungen.

Die Kompositionsglieder der untersuchten WBK bestehen in zahlreichen Fällen aus simplizischen Einheiten. Sonst ist für sie ein Reichtum der Wortbildungsarten kennzeichnend: Vor allem kann eine Dominanz der Suffixderivation festgestellt werden, während implizite Derivation, Rückbildung, Konversion und Wortkreuzung zu den seltenen Wortbildungsarten gehören.

Die Beurteilung der Produktivität gehört nicht zu den deklarierten Zielen dieses Beitrags, doch tauchten im Laufe der Untersuchung Indizien wie hohe Typenzahl und großer Anteil der Okkasionalismen auf, die auf eine hohe Produktivität der -fritze-Bildungen schließen lassen, aber auch solche (hohe Tokenzahl nur bei wenigen Lexemen), die dagegensprechen. Es bedarf noch gründlicherer Untersuchungen und der Berücksichtigung mehrerer Aspekte, um darüber stichhaltige Aussagen machen zu können. ${ }^{35}$

Im Rahmen dieser Studie war es nicht möglich, uns mit Fragen der Fugengestaltung und der Rechtschreibung auseinanderzusetzen. Eine Fortführung der Forschung in diese Richtung ist geplant.

Eine kontrastive semantische Untersuchung von WBK mit den deonymischen Vornamen -liese und -fritze und ihren ungarischen Entsprechungen erscheint in Kürze (vgl. Harsányi demn.).

Zukünftige Forschungsmöglichkeiten bieten weitere Wortbildungsmodelle mit appellativischen Vornamen und diejenigen grammatischen, semantischen und lexikongebundenen Beschränkungen, ${ }^{36}$ denen diese unterliegen. ${ }^{37}$

35 Vgl. dazu Fleischer/Barz (2012: 75): „Hochproduktiv [...] sind Modelle dann, wenn sie nur wenige Restriktionen in Bezug auf phonologische, morphologische, syntaktische und semantische Eigenschaften des Inputs aufweisen (qualitatives Kriterium) und in hohem Maße auch für Neubildungen genutzt werden (quantitatives Kriterium)".

36 S. Fleischer/Barz (2012: 79).

37 Bergmann (1971: 106) untersucht in seiner Studie, welche Bedingungen ein Verb erfüllen muss, um appellativische Bildungen auf -fritze einzugehen. Er kommt dabei zu der folgenden Schlussfolgerung: „Es muß sich um menschliche Tätigkeiten handeln, die tadelbar (aber nicht zu schwerwiegend) sind, bei denen eine gewisse Aktivität vorhanden ist und die willentlich wiederholbar ist, ohne daß sie auf ein Ziel (oder einen Partner) gerichtet sind. Die Verben dürfen stilistisch nicht zu hoch sein, und sie müssen der syntagmatischen Forderung genügen, daß sie im Satz ohne Bindungen stehen können." 


\section{Literatur}

\subsection{Quellen}

Das Deutsche Referenzkorpus DeReKo, am Leibniz-Institut für Deutsche Sprache, Mannheim. http://www.ids-mannheim.de/kl/projekte/korpora (abgerufen am 17.03.2020).

\subsection{Sekundärliteratur}

Bergmann, Gunter (1971): Zur Theorie der Wortbildungsregeln. (Der Typ „Heulsuse") In: Deutsch als Fremdsprache (8). S. 104-108.

Donalies, Elke (2002): Die Wortbildung des Deutschen. Ein Überblick. Tübingen: Gunter Narr Verlag.

Eisenberg, Peter (2013): Grundriss der deutschen Grammatik: Band 1: Das Wort. Stuttgart/Weimar: Verlag J. B. Metzler. https://doi.org/10.1007/978-3476-00743-8_1

Elsen, Hilke (2009): Affixoide: Nur was benannt wird, kann auch verstanden werden. In: Deutsche Sprache (37), S. 316-333. https://doi.org/10.37307/ j.1868-775X.2009.04.03

Fleischer, Wolfgang/Barz, Irmhild (1995): Wortbildung der deutschen Gegenwartssprache. Tübingen: Max Niemeyer Verlag.

Fleischer, Wolfgang/Barz, Irmhild (2012): Wortbildung der deutschen Gegenwartssprache. Berlin: de Gruyter. https://doi. org/10.1515/9783110256659

Harsányi, Mihály (demn.): Zur Semantik von Wortbildungskonstruktionen mit deonymischen Vornamen als Letztglied im Deutschen und Ungarischen. Innsbruck.

Henzen, Walter (1965): Deutsche Wortbildung. Tübingen: Max Niemeyer.

Herberg, Dieter/Kinne, Michael/Steffens, Doris (2004): Neuer Wortschatz: Neologismen der 90er Jahre im Deutschen. Berlin/New York: de Gruyter. https://doi.org/10.1515/9783110902273

Ortner, Lorelies [u. a.] (1991): Substantivkomposita. Berlin/New York: de Gruyter. https://doi.org/10.1515/9783110847628

Schneider-Wiejowski, Karina (2011): Produktivität in der deutschen Derivationsmorphologie. Dissertation. Bielefeld: Universitätsbibliothek Bielefeld. 


\subsection{Lexika}

DBW - Duden. Das Bedeutungswörterbuch. Hg. von der Dudenredaktion. Mannheim, Leipzig, Wien, Zürich: Dudenverlag, 2010.

DHW - Duden. Das Herkunftswörterbuch. Etymologie der deutschen Sprache. Hg. vom Wiss. Rat d. Dudenredaktion. Mannheim, Wien, Zürich: Dudenverlag, 1989.

Do - Duden online. https://www.duden.de (abgerufen am 15.06.2020).

DR - Duden. Die deutsche Rechtschreibung. Hg. von der Dudenredaktion. Mannheim, Leipzig, Wien, Zürich: Dudenverlag, 2010 [CD-Rom].

DS - Duden. Das Synonymwörterbuch. Hg. von der Dudenredaktion. Mannheim: Bibliographisches Institut, 2004 [CD-Rom].

DUW - Duden. Deutsches Universal Wörterbuch. Hg. von der Dudenredaktion. Mannheim: Bibliographisches Institut, 2011 [CD-Rom].

DW - Deutsches Wörterbuch von Jacob Grimm und Wilhelm Grimm, Erstbearbeitung (1854-1960), digitalisierte Version im Digitalen Wörterbuch der deutschen Sprache. https://www.dwds.de/wb/dwb (abgerufen am 15.06.2020).

DWDS - Digitales Wörterbuch der deutschen Sprache. https://www.dwds.de (abgerufen am 15.06.2020).

LDaF - Langenscheidts Großwörterbuch Deutsch als Fremdsprache. Hg. von Dieter Götz/Günther Haensch/Hans Wellmann. Mannheim 1999 [CD-Rom].

Pfeifer, Wolfgang [u.a.] (1993): Etymologisches Wörterbuch des Deutschen, digitalisierte und von Wolfgang Pfeifer überarbeitete Version im Digitalen Wörterbuch der deutschen Sprache. https://www.dwds.de/wb/etymwb (abgerufen am 14.06.2020). 


\section{Anhang}

Wortbildungskonstruktionen auf -fritze, nach Häufigkeit absteigend. In Klammern: Tokenzahl.

Werbefritze/Werbe-Fritze/Werbefritze (245)

Pressefritze/Presse-Fritze (114)

Fernsehfritze/Fernseh-Fritze (89)

Meckerfritze (88)

Zeitungsfritze (88)

Radiofritze/Radio-Fritze/RadioFritze

Filmfritze/Film-Fritze (54)

Marketingfritze/Marketing-Fritze/

Markeding-Fritze (46)

Reklamefritze/Reklame-Fritze (36)

Computerfritze/Computer-Fritze (27)

Versicherungsfritze (26)

Medienfritze/Medien-Fritze (25)

Öko-Fritze/Ökofritze (23)

Immobilienfritze/Immobilien-Fritze

(22)

PR-Fritze (19)

Feuerfritze/Feuer-Fritze (17)

Nörgelfritze (17)

Fahrradfritze/Fahrrad-Fritze/

Fahradfritze (16)

Modefritze (16)

Plapperfritze (13)

Stänkerfritze (13)

Kinofritze (12)

Verkehrsfritze (12)

Asta-Fritze/AStA-Fritze (10)

Autofritze/Auto-Fritze (10)

Königsfritze (10)

Mäkelfritze (10)

Psycho-Fritze/Psychofritze (10)

Flaschenfritze (9)

Parteifritze (9)

Theaterfritze/Theater-Fritze/

Theatafritze (9)

Fischfritze (8)

TV-Fritze (8)
Kultusfritze (7)

SED-Fritze (7)

Techno-Fritze/Technofritze (7)

Teerfritze (7)

Biofritze/Bio-Fritze/biofritze (6)

Gemüsefritze/Jemüsefritze (6)

Kunstfritze (6)

Zauber-Fritze/Zauberfritze (6)

Agenturfritze (5)

Atomfritze (5)

Bankfritze (5)

CDU-Fritze (5)

Finanzfritze (5)

Internetfritze/Internet-Fritze (5)

Kirchenfritze (5)

Monokelfritze (5)

Pingelfritze (5)

Stasi-Fritze/Stasifritze (5)

Baufritze (4)

Comedy-Fritze (4)

Fotofritze/Photofritze (4)

Google-Fritze/google-Fritze/

Googlefritze (4)

Moralfritze (4)

Pharma-Fritze/Pharmafritze (4)

Politfritze/Polit-Fritze (4)

Sabbelrritze/Sabbelfritze (4)

Schlagerfritze/Schlager-Fritze (4)

Steuerfritze (4)

Telefonfritze (4)

Umweltfritze/Umwelt-Fritze (4)

Verwaltungsfritze (4)

Bierdeckel-Fritze/Bierdeckelfritze (3)

EU-Fritze (3)

Feuilletonfritze (3)

Fußballfritze (3)

Gewerkschaftsfritze (3)

Hollywood-Fritze (3)

IT-Fritze (3) 
Kamerafritze (3)

Lächelfritze (3)

Lotto-Fritze/Lottofritze (3)

MiniFritze (3)

Möbelfritze (3)

Obstfritze (3)

Ost-Fritze/Ostfritze (3)

Pizzafritze (3)

Plattenfritze (3)

Postfritze (3)

Pöbelfritze (3)

Schlossfritze (3)

Schrottfritze (3)

Showfritze (3)

Sport-Fritze/Sportfritze (3)

Tourismus-Fritze (3)

Verbandsfritze (3)

Verschwörungsfritze/

Verschwoerungsfritze (3)

Wetterfritze (3)

Witzefritze (3)

AfD-Fritze (2)

Anti-Kult-Fritze (2)

Antiquitätenfritze (2)

Astrofritze (2)

Bibel-Fritze/Bibelfritze (2)

Bouletten-Fritze/Boulettenfritze (2)

Business-Fritze/Businessfritze (2)

Butterfritze (2)

Bürofritze (2)

Champagnerfritze (2)

Chemiefritze (2)

CIA-Fritze (2)

Comicfritze (2)

Dorffritze (2)

EP-Fritze (2)

Event-Fritze (2)

FDP-Fritze (2)

Feng-Shui-Fritze (2)

Fifa-Fritze (2)

Flughafenfritze (2)

«Gault Millau»-Fritze (2)

Geldfritze (2)

Gesellschaftsfritze (2)
Getränkefritze (2)

Handy-Fritze/Handyfritze (2)

Heulfritze (2)

Hollywood-Nightclubfritze (2)

Hotelfritze (2)

Impeachment-Fritze (2)

Käferfritze (2)

Kartenfritze (2)

Käsefritze (2)

Klamauk-Fritze/Klamaukfritze (2)

Kleckerfritze (2)

Klempner-Fritze (2)

Klimafritze (2)

Klüngel-Fritze/Klüngelfritze (2)

Kohlenfritze (2)

Kokelfritze (2)

Kommunikationsfritze (2)

Lederfritze (2)

Manschetten-Fritze (2)

Musikfritze (2)

Naturschutzfritze (2)

Nazi-Fritze (2)

News-Fritze (2)

NPD-Fritze (2)

Olympia-Fritze (2)

ÖV-Fritze (2)

Paketfritze (2)

Pegida-Fritze/'Pegida'-Fritze (2)

Planerfritze (2)

Polizeifritze (2)

Pop-Fritze (2)

Preußen-Fritze/preußen-fritze (2)

Radsportfritze (2)

Regierungsfritze (2)

Reisebüro-Fritze/Reisebürofritze (2)

Rundfunkfritze (2)

Schlaufritze (2)

Schnapsfritze (2)

Schwitze-Fritze/Schwitzefritze (2)

Security-Fritze/Securityfritze (2)

Senatsfritze (2)

SF-Fernsehfritze (2)

Sicherheitsfritze (2)

Skifritze (2) 
Soldatenfritze (2)

Spurenfritze (2)

Stöcklschuhfritze (2)

Tageszeitungsfritze (2)

Templerfritze (2)

Teppichfritze (2)

Trolleybus-Magazine-Fritze (2)

TV-Versicherungsfritze (2)

UFO-Fritze (2)

Unterhaltungsfritze (2)

Veranstaltungsfritze (2)

Vereinsfritze (2)

Verlagsfritze (2)

Vermarktungsfritze (2)

Video-Fritze/Videofritze (2)

Wanderfritze (2)

Wessi-Fritze (2)

Wuselfritze (2)

Zahlenfritze (2)

Zigarettenfritze (2)

Zigarrenfritze (2)

Zollfritze (2)

Absatz-Oberfritze (1)

Allianz-Fritze (1)

Almdudler-Fritze (1)

Amifernsehfritze (1)

Andenkenfritze (1)

Angelfritze (1)

Animierfritze (1)

Anlagenfritze (1)

Antriebsfritze (1)

Anzeigenfritze (1)

Anzugfritze (1)

Anzug-und-Krawatte-Fritze (1)

Aufsichtsfritze (1)

Ausbildungsfritze (1)

Ausdauerfritze (1)

Aussenhandelsfritze (1)

Automarketingfritze (1)

Automatenfritze (1)

Babbelfritze (1)

Bankenfritze (1)

Barkassenfritze (1)

Baumarkt-Fritze (1)
Beamtenfritze (1)

Betonfritze (1)

Bettelfritze (1)

Bettenfritze (1)

Bezirksfritze (1)

BfN-Fritze (1)

Biedermeierfritze (1)

Bierfritze (1)

Bilderfritze (1)

Bild-Fritze (1)

Bildungsfritze (1)

Billigfritze (1)

BMW-Fritze (1)

Boulevardfritze (1)

Börsenfritze (1)

Broilerfritze (1)

Buchfritze (1)

Bürokratenfritze (1)

Chansonfritze (1)

Cocacola-Fritze (1)

Coca-Cola-Werbefritze (1)

Country-Fritze (1)

CS-Fritze (1)

Datenverarbeitungsfritze (1)

Designfritze (1)

Deutsche-Welle-Fritze (1)

Devisenfritze (1)

Discounter-Fritze (1)

Diskofritze (1)

Drängelfritze (1)

Drehbuch-Fritze (1)

Dürer-Fritze (1)

Elektrofritze (1)

Energiefritze (1)

Entscheidungsfritze (1)

Erdkundefritze (1)

E-Roller-Fritze (1)

Ersatzteilfritze (1)

Esoterikfritze (1)

Ethikfritze (1)

Europa-Fritze (1)

Europa-Werbefritze (1)

Ex-Asta-Fritze (1)

Expo-Fritze (1) 
Express-Fritze (1)

Ex-Werbefritze (1)

Facebookfritze (1)

Fachfritze (1)

Faschingsfritze (1)

Faxenfritze (1)

FDGB-Fritze (1)

FDP-Wirtschaftsfritze (1)

Feierfritze (1)

Ferienwohnung-Fritze (1)

Fiction-Fritze (1)

Filmförderungskommissionsfritze (1)

Finanzkrisen-Fritze (1)

Flakfritze (1)

Flötenfritze (1)

Flugsicherungs-Fritze (1)

FPÖ-Werbefritze (1)

Fremdenverkehrs-Fritze (1)

Fusions-Fritze (1)

Fußball-Verbands-Fritze (1)

Fusselbartfritze (1)

Gebrauchtwagenfritze (1)

Gehirnfritze (1)

Gemeindefritze (1)

Gemischtwarenfritze (1)

Genderfritze (1)

Geschenkpapier-Bastelfritze (1)

Getränkemarktfritze (1)

Gierfritze (1)

Gitarrenfritze (1)

GPS-Werbefritze (1)

Greenpeace-Fritze (1)

Grün-Fritze (1)

Grünkramfritze (1)

Gugelfritze (1)

Gurkenfritze (1)

Gyros-Fritze (1)

Hähnchen-Fritze (1)

Halbstadtfritze (1)

Hamburger-Fritze (1)

Handbuchfritze (1)

Hardcore-Fritze (1)

Heinkel-Fritze (1)

Hemdenfritze (1)
Hollywood-Filmmusikfritze (1)

Homecomputerfritze (1)

Horrorfritze (1)

Hundefritze (1)

Infofritze (1)

Infotainment-Fritze (1)

Investmentfritze (1)

Jagdfritze (1)

Jammerfritze (1)

Jauche-Fritze (1)

Jodelfritze (1)

Journalisten-Fritze (1)

Kabelfernsehfritze (1)

Kabelfritze (1)

Kachelofenfritze (1)

Kaltwaren-Marketingfritze (1)

Kartonagenfritze (1)

KB-Fritze (1)

Kicherfritze (1)

Kinderbücherfritze (1)

Klassikfritze (1)

Kletterfritze (1)

Knallfritze (1)

Knorr-Fritze (1)

Kommunalfritze (1)

Kraftstoff-Fritze (1)

Krawall-Fritze (1)

Kripo-Fritze (1)

Künstlerfritze (1)

Laberfritze (1)

Länderfritze (1)

Landkreis-Fritze (1)

Ländlerfritze (1)

Landwirtschaftsfritze (1)

Landwirtschaftskammer-Fritze (1)

Law-andorder-Fritze (1)

Lebensberatungsfritze (1)

Limonaden-Fritze (1)

Lotto-Toto-Fritze (1)

Marinefritze (1)

Marionettenfritze (1)

Maschinenfritze (1)

Messefritze (1)

Modehaus-Fritze (1) 
Moped-Fritze (1)

Musicalfritze (1)

Musikwirtschaftswerbefritze (1)

Müllfritze (1)

Müsli-Fritze (1)

Nähmaschinenfritze (1)

New-Age-fritze (1)

Nightclubfritze (1)

Nobelfritze (1)

Notenfritze (1)

NS-Fritze (1)

OECD-Bildungsfritze (1)

Opernfritze (1)

Organisations-Fritze (1)

Orgelfritze (1)

Ortsartikelfritze (1)

Öl-Fritze (1)

Paläo-Diät-Fritze (1)

Paläofritze (1)

Panikfritze (1)

Papierfritze (1)

Parfümfritze (1)

Parkplatzfritze (1)

Personalfritze (1)

Pfaffenfritze (1)

Pfandhaus-Fritze (1)

Pfefferfritze (1)

Pimmelfritze (1)

Pinselfritze (1)

Piratenfritze (1)

Pizzen-Fritze (1)

Plagifritze (1)

Plauderfritze (1)

Polit-Werbefritze (1)

Pommesbuden-Laberfritze (1)

Porno-Fritze (1)

Porzellanfritze (1)

Post-\&-Telekom-Werbefritze (1)

Programmfritze (1)

Projektfritze (1)

Public-Relations-Fritze (1)

Pulver-Fritze (1)

Rackerfritze (1)

Randalefritze (1)
Rätselfritze (1)

Rechenzentrums-Fritze (1)

Reformfritze (1)

Regiefritze (1)

Reifenfritze (1)

Reisefritze (1)

Reparaturfritze (1)

Reporterfritze (1)

Rockfritze (1)

Rosenfritze (1)

Routine-Fritze (1)

Röntgenfritze (1)

Rubbelfritze (1)

Rummelfritze (1)

Sammelfritze (1)

Schallplattenfritze (1)

Schickimicki-Fritze (1)

Schmierfritze (1)

Schoggifritze (1)

Schriftstellerfritze (1)

Schuhfritze (1)

Schwafelfritze (1)

SD-Fritze (1)

Securitas-Fritze (1)

Sekten-Fritze (1)

Selbstdarstellungsfritze (1)

Senffritze (1)

Sensationsfritze (1)

Sexfilm-Fritze (1)

Signalfritze (1)

Skoliosefritze (1)

Solarium-Fritze (1)

Sony-Fritze (1)

Spaghetti-Fritze (1)

Sparkassen-Fritze (1)

Spektakelfritze (1)

Spiegelfritze (1)

Sponsorfritze (1)

Staatsschutzfritze (1)

Stadtmarketingfritze (1)

Start-up-Fritze (1)

Steuermodell-Fritze (1)

Stimmungsfritze (1)

Stotterfritze (1) 
Störenfritze (1)

Strampelfritze (1)

Stromfritze (1)

Studiofritze (1)

Suppen-Fritze (1)

SUV-Fritze (1)

Szenefritze (1)

Tabakfritze (1)

Tabellenfritze (1)

Talkshowfritze (1)

Tankwartsfritze (1)

Tattoo-Fritze (1)

TdS-Felgenfritze (1)

Technikfritze (1)

Technischer-Überwachungs-Fritze (1)

Telefritze (1)

Tennisfritze (1)

Terrornoise-Fritze (1)

T-Fritze (1)

Tintenfritze (1)

T-Online-Fritze (1)

Torpedofritze (1)

Toto-Fritze (1)

Tranfritze (1)

TV-Werbefritze (1)

Uefa-Fritze (1)

Umfrage-Fritze (1)

Unions-Fritze (1)

UNMIK-Fritze (1)

UNO-Fritze (1)

Upperclass-Fritze (1)

Urheberrechtsfritze (1)

Verbesserungsfritze (1)

Verbindungsfritze (1)

Vergasungsfritze (1)

Versorgungsfritze (1)

Verteidigungsfritze (1)

Vertreter-Fritze (1)

Videoladen-Fritze (1)

Vitaminfritze (1)

Vodafone-Werbefritze (1)

Vogelfritze (1)

Wald- und Wiesen-Pressefritze (1)

Wäschefritze (1)
Waschmaschinenfritze (1)

Wasserfritze (1)

Weinfritze (1)

West-Pressefritze (1)

Wettbewerbsfritze (1)

Wettbürofritze (1)

Whisky-Fritze (1)

Wikipedia-Fritze (1)

Wirtschafts-Fritze (1)

Wissenschaftsfritze (1)

Witzhemdchenfritze (1)

Wurstfritze (1)

Zahnpastafritze (1)

Zappelfritze (1)

ZDF-Frühstücksfernsehfritze (1)

Zementfritze (1)

Zoo-Fritze (1)

Zündelfritze (1)

Zweiradfritze (1)

IG-Metall-Fritze (1)

Imbissbudenfritze (1)

Inkasso-Fritze (1)

Jazz-Fritze (1)

Jetset-Fritze (1)

Jubelfritze (1)

Kara-Möbelfritze (1)

Kartoffelfritze (1)

Kfz-Fritze (1)

Konsumfritze (1)

Konzertfritze (1)

Kosmetikfritze (1)

Kräuterfritze (1)

Kultur-Info-Fritze (1)

Lackfritze (1)

Lamettafritze (1)

Macherfritze (1)

Mährfritze (1)

Mallorca-Fritze (1)

Mathefritze (1)

Mietwagenfritze (1)

Maschinenbaufritze (1)

Multikulti-Fritze (1)

Muschel-Fritze (1) 